\title{
Comparison of the First Order and the Second Order Equivalent Circuit Model Applied in State of Charge Estimation for Battery Used in Electric Vehicles
}

\author{
Guoliang Wu ${ }^{1}$, Chunbo Zhu ${ }^{2}$, and C. C. Chan ${ }^{3}$ \\ ${ }^{1}$ School of Electrical Engineering, Harbin Institute of Technology,wuguolianghit@gmail.com \\ ${ }^{2}$ School of Electrical Engineering, Harbin Institute of Technology, zhuchunbo@hit.edu.cn \\ ${ }^{3}$ School of Electrical Engineering, Harbin Institute of Technology, ccchan@eee.hku.hk
}

\begin{abstract}
In this study, the first order model and the second order equivalent circuit models of NiMH battery for electric vehicle were used to determinate the battery state of charge. Different parameters for equivalent circuit models were explained. There are four phases in the battery change and discharge, which are charge phase, change idle phase, discharge phase, discharge idle phase. All of these phases are discussed and analyzed. For different phases of charge and discharge, mathematical relationships between parameters and state of charge were built. The calculation results fit well with the experimental results. A precision comparison between the first order model and the second order model was undertaken.
\end{abstract}

\section{Keywords}

electric vehicles, NiMH battery, equivalent circuit models, state of charge, model parameters

\section{INTRODUCTION}

State of charge (SOC) represents the residual capacity of the battery and it is written as the percent of residual capacity by nominal capacity. The estimation of SOC of NiMH battery is a key point of energy management system in EV/HEV. With accurate SOC, the battery can extend its age and avoid being over charged or over discharged [Jung et al., 2002]. Moreover it is benefit for controller to implement control strategy in HEV. Many methods are used to improve the precision of SOC.

It is very popular to estimate the SOC with Ampere hours (Ah) algorithm, which apply in EV/HEV. The battery capacity is measured in Ah algorithm which is calculated by multiplying the current by time of discharge [Koehler et al., 2001, Stiegeler et al., 2005]. The residual capacity is calculated by initial capacity minus capacity discharged. Researcher found it is more precise when it considers the compensation, such as temperature, discharge rate, self discharge and age [Youn et al., 2005]. But Ah algorithm has three shortcomings. First, it is impossible to forecast the initial SOC. Second its estimation precision is dependent on the precision of sensor. Third, the accumulated error can not be ignored with the test time growing. For longer time periods and operation where incomplete charging is concerned, Ah-balancing results in high errors [Kremer et al., 1995, Terry and Wang, 2005, Ha et al., 1992].
Open circuit Voltage method is widely used in estimation initial SOC of the battery. The terminal Voltage of the battery is relevant with the SOC when the battery is under no load, or the battery is static. Therefore, before EV/HEV running, the battery management system measures the battery terminal Voltage in order to evaluate the battery SOC. However, in the battery charge or discharge state, the battery is not in open circuit condition, which leads SOC is inaccurate by open circuit Voltage method. The most important thing for open circuit Voltage method is that the battery has to be idle for more than ten hours to be steady. Open circuit Voltage method is hard to meet this request for EV/HEV. It is popular to use open circuit Voltage method and Ah algorithm together to achieve the SOC [Onishi et al., 2001].

Neural network establishes a relationship between input and output data, which uses Voltage, current, temperature as its input and the SOC as output. In order to train this artificial neural network based model, the data were collected after a series of the designed experiments were carried out using the battery evaluation and testing system with the wide range of discharge current and temperature [Singh and Reisner, 2002, Chan et al., 2000]. The virtue of the method is it can be applied to most the battery systems. However, training data affects the SOC strongly.

A correlation between electrical parameters, extracted directly from the high frequency region of ac impedance spectra is found out in laboratory [Abderrezak et al., 2004]. In paper [Salkind et al., 1999], a method involves the use of fuzzy logic mathematics to analyze data obtained by impedance spectroscopy and 
coulomb counting techniques. A fuzzy logic model is developed by measuring the impedance at three frequencies data to estimate SOC [Singh et al., 2004]. But impedance spectra measure is expensive to implement in EV/HEV.

In paper [Barbarisi et al., 2002], the SOC is estimated by computing the dynamic behavior of the $\mathrm{Ni}(\mathrm{OH})_{2}$ concentrations in the active nickel-hydroxide film and using measurements of current and Voltage. However, a key factor is that it is hard to study dynamic behavior $\mathrm{Ni}(\mathrm{OH})_{2}$ of in the battery electrode when the battery is fixed in EV/HEV.

In this paper, a SOC estimation method based on the battery equivalent circuit models is proposed, which sets a relationship between the battery SOC and electromotive force and sets a relationship among SOC, the battery terminal Voltage and parameters of equivalent circuit by experiments. Thus SOC can be calculated by the battery terminal Voltage and parameters of equivalent circuit. In this paper, not only the second order equivalent circuit is introduced, but also the first order equivalent circuit is discussed. Moreover, four phases of charge and discharge of the battery equivalent circuit are analyzed in detail. In addition, the NiMH battery is used to verify the precision of equivalent circuit models.

\section{THE RELATIONSHIP OF NIMH BATTERY SOC AND ELECTROMOTIVE FORCE}

To determinate the relationship of $\mathrm{NiMH}$ battery SOC and electromotive force (EMF), a pulse discharge experiment was designed. The battery was 10 $\mathrm{Ah} / 1.2 \mathrm{~V}$. The procedure includes discharge pulse discharge experiment and pulse charge experiment. For pulse discharge experiment, first, NiMH battery was charged fully and idled till it was steady. Second, the battery discharged $1 \mathrm{Ah}$ each time, there were 10 times in an experiment that insured SOC decreased 0.1 . It idled enough long time between two pulses to ensure the battery was in steady condition. Then we recorded the 11 open circuit Voltages after each pulse discharge (OCVd) under different SOC. In the same way, for pulse discharge experiment, first, NiMH battery was discharged to empty state and idled till it was steady. Second, the battery charged 1 Ah each time, there were 10 times in an experiment that insured SOC increased 0.1. It idled enough long time between two pulses to ensure the battery was in steady condition. Then we recorded the 11 open circuit Voltages after each pulse charge $(\mathrm{OCVc})$ under different SOC. The average value of open circuit Voltage after pulse charge and open circuit Voltage after pulse discharge equals to electromotive force (EMF) approximately. The relationship of SOC and EMF shows in Figure

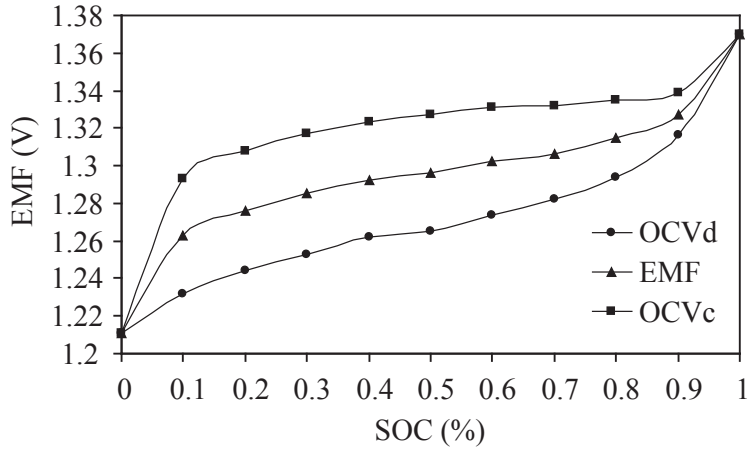

Fig. 1 The relationship between electromotive force and state of charge

1. It shows a good linear relationship at SOC 0-0.1, $0.2-0.8,0.8-1$ parts. Therefore a formula has been proposed in equation (1), and $a_{i}, b_{i}(i=1,2,3)$ are coefficients determined by experiment.

$=\left\{\begin{array}{ll}a_{1} \varepsilon+b_{1} & 0-0.1 \\ a_{2} \varepsilon+b_{2} & 0.1-0.8 \\ a_{3} \varepsilon+b_{3} & 0.8-1\end{array}\right\}$

$\theta$ represents SOC, $a_{i}$ and $b_{i}$ ( $i$ equals $1,2,3$ ) are coefficients determined by experiment data. $\varepsilon$ is electromotive force.

\section{THE FIRST ORDER MODEL ANALYSIS AND SOC ESTIMATION}

The battery internal resistance includes ohm resistance and polarization resistance. In Figure 2, it shows a first order NiMH battery model. $R_{\Omega}$ represents ohm resistance, $R_{k d}$ represents polarization resistance, $C_{k d}$ is parallel capacitor, $V_{t}$ is terminal Voltage, $\varepsilon$ is EMF. $\tau_{k d}$ is time constant whose value is $R_{k d} C_{k d} . V_{k d 0}$ is initial value of $C_{k d}$ when the battery is in charge or discharge. $I$ represents charge/discharge current.

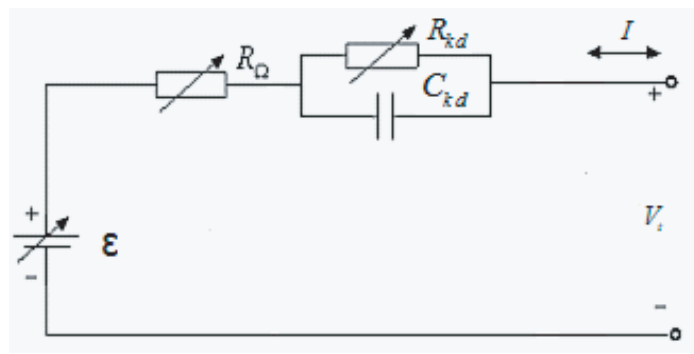

Fig. 2 The first order equivalent circuit model

\subsection{Discharge process}

When the battery begins to discharge, the battery polarization appears. $C_{k d}, R_{k d}$ branch circuit is under zero state response at the current switching on moment. Then $C_{k d}$ begins to accumulate charge. $C_{k d}, R_{k d}$ branch circuit equation shows in (2). The battery terminal 
Voltage formula shows in (3). $V_{k d}$ represents terminal Voltage of $C_{k d}$.

$R_{k d} C_{k d} \frac{\mathrm{d} V_{k d}}{\mathrm{~d} t}+V_{k d}=I(t) R_{k d}$

$V_{t}=\varepsilon-I(t) R_{\Omega}-V_{k d}$

$V_{k d}$ equals $I(t) R_{k d}\left(1-\mathrm{e}^{-t / \tau_{k d}}\right)$ by solving equation (2). $\tau_{k d}$ is time constant, whose value is $R_{k d} C_{k d}$. Put $V_{k d}$ and (3) into (1), SOC formula can be achieved, which shows in (4).

$\theta=a_{i} V_{t}+a_{i} I(t) R_{\Omega}+a_{i} I(t) R_{k d}\left(1-\mathrm{e}^{-t / \tau_{k d}}\right)+b_{i}$

\subsection{Discharge idle process}

When the battery stops discharge, the battery polarization begins to decrease. $C_{k d}, R_{k d}$ branch circuit is under zero input response at the current switching off moment. There is no current who flows through $R_{\Omega}$. $C_{k d}$, $R_{k d}$ branch circuit equation shows in (5). The battery terminal Voltage formula shows in (6). SOC formula shows in (7). $V_{k d}$ equals $V_{k d 0} \mathrm{e}^{-t / \tau_{k d}}, V_{k d 0}$ is the initial Voltage of $C_{k d}$.

$R C_{k d} C_{k d} \frac{\mathrm{d} V_{k d}}{\mathrm{~d} t}+V_{k d}=0$

$V_{t}=\varepsilon-V_{k d}$

$\theta=a_{i} V_{t}+a_{i} V_{k d 0} \mathrm{e}^{-t / \tau_{k d}}+b_{i}$

\subsection{Charge phase analysis}

The analysis of charge phase is similar with discharge phase. For charge process, formula of SOC shows in (8) and the value of $V_{k d}$ is $I(t) R_{k d}\left(1-\mathrm{e}^{-t / \tau k d}\right)$. For charge idle process, SOC formula shows in (9), $V_{k d}$ equals $V_{k d 0} \mathrm{e}^{-t / \tau k d}$.

$\theta=a_{i} V_{t}-a_{i} I(t) R_{\Omega}-a_{i} I(t) R_{k d}\left(1-\mathrm{e}^{-t / \tau_{k d}}\right)+b_{i}$

$\theta=a_{i} V_{t}-a_{i} V_{k d 0} \mathrm{e}^{-t / \tau_{k d}}+b_{i}$

\section{SECOND ORDER MODEL ANALYSIS AND SOC ESTIMATION}

The difference of the second order model and the order model is polarization resistance. For second order model, polarization resistance is divided into electrochemical polarization resistance and diffusion resistance. Second order NiMH battery model shows in Figure 3. $R_{k}$ represents polarization resistance, $R_{d}$ represents electrochemical polarization. $C_{k}, C_{d}$ are parallel capacitors with $R_{k}$ and $R_{d}$. For second order

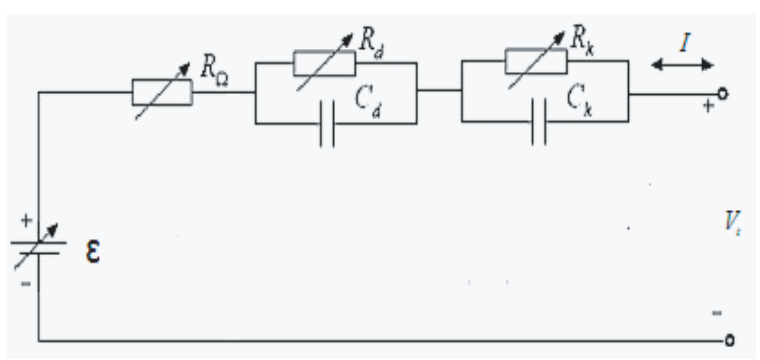

Fig. 3 Second order equivalent circuit model

model, SOC formula is shown in (10). $\tau_{k}$ and $\tau_{d}$ are time constants, whose value are $R_{k} C_{k}$ and $R_{d} C_{d}$ respectively. $V_{k 0}$ and $V_{d 0}$ are initial values of $C_{k}$ and $C_{d}$ under the battery charge/discharge idle state.

$\theta= \begin{cases}a_{i}\left(V_{t}+I(t) R_{\Omega}+V_{k}+V_{d}\right)+b_{i} & \text { discharge } \\ a_{i}\left(V_{t}+V_{k}+V_{d}\right)+b_{i} & \text { discharge idle } \\ a_{i}\left(V_{t}-I(t) R_{\Omega}-V_{k}-V_{d}\right)+b_{i} & \text { charge } \\ a_{i}\left(V_{t}-V_{k}-V_{d}\right)+b_{i} & \text { charge idle }\end{cases}$

where,

$V_{k}= \begin{cases}I(t) R_{k}\left(1-\mathrm{e}^{-t / \tau_{k}}\right) & \text { discharge idle } \\ I(t) R_{k}\left(1-\mathrm{e}^{-t / \tau_{k}}\right) & \text { discharge idle }\end{cases}$

$V_{d}= \begin{cases}I(t) R_{d}\left(1-\mathrm{e}^{-t / \tau_{d}}\right) & \text { discharge or charge } \\ V_{d 0} \mathrm{e}^{-t / \tau_{\mathrm{d}}} & \text { discharge idle or charge idle }\end{cases}$

\section{PARAMETERS IDENTIFICATION OF EQUIVALENT CIRCUIT MODE}

\subsection{Experiment object, equipment and process}

This experiment used a $10 \mathrm{Ah} / 1.2 \mathrm{~V} \mathrm{NiMH}$ battery and it was tested by Arbin BT2000 the battery test instrument. The battery test system for the experiment is shown in Figure 4, which includes data acquisition card, computer, NiMH battery. The battery Voltage and current were measured by sensors of Arbin BT2000 and then their values were stored in computer. Each precise of Voltage and current is $0.1 \%$. During the process of measuring the battery Voltage and current, data were recorded in an excel file auto-

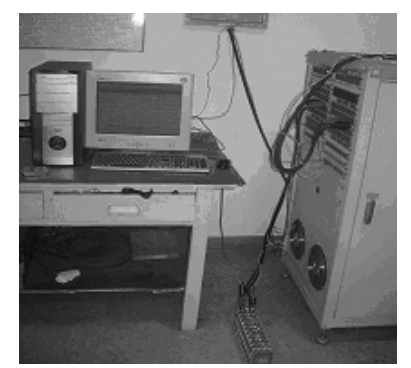

Fig. 4 The battery test system 
matically. This monitor system can show current and Voltage curves in real time. Before data acquisition, sample time and save file path have to be set.

In this discharge experiment, the battery was discharged by pulse current. Experiment was undertaken when NiMH battery was in full charge state. The discharge current was $5 \mathrm{~A}$. The battery discharged $1 \mathrm{Ah}$ every time, there were 10 times in an experiment that insures SOC decreases 0.1. It idles several times between two pulses to ensure the battery is in steady condition. The experiment shows in Figure 5.

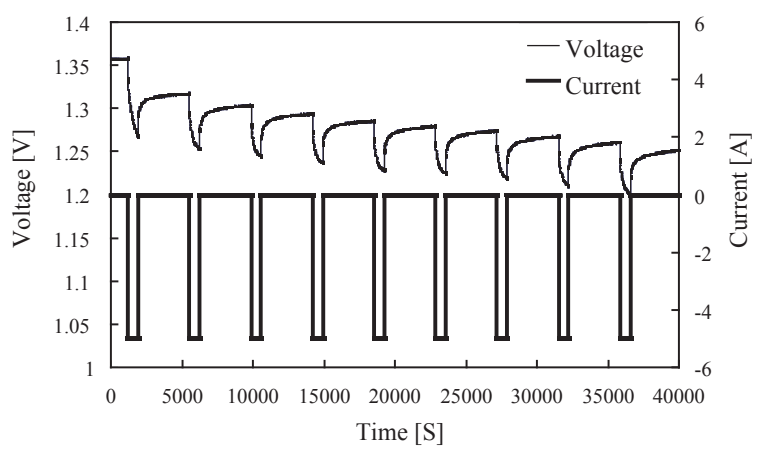

Fig. 5 NiMH battery pulse discharge curve

In this charge experiment, the battery was charged by pulse current. Experiment was undertaken when NiMH battery was in empty state. The charge current was $5 \mathrm{~A}$. The battery charged $1 \mathrm{Ah}$ every time, there were 10 times in an experiment that insures SOC decreases 0.1 . It idles several times between two pulses to ensure the battery is in steady condition. The experiment shows in Figure 6.

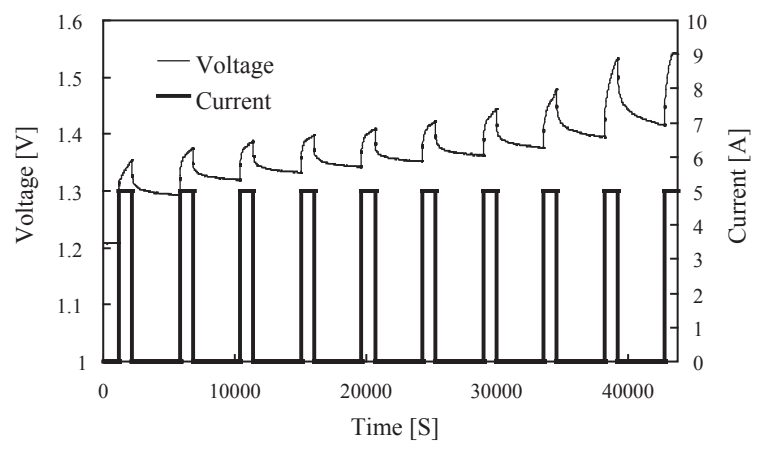

Fig. 6 NiMH battery pulse charge curve

\subsection{Parameters identification}

$V_{t}$ and $I$ are measured by Voltage sensor and current sensor. From the experiment data, $R_{\Omega}, R_{k d}, R_{d}, R_{k}$ and $\tau_{k d}, \tau_{d}, \tau_{k}$ are identified. $R_{\Omega}$ is achived by experiment. Ohm internal resistance use DC method. $R_{k d}, R_{d}, R_{k}$ are identified by curve Least-squares fitting with experiment data, which use the method in reference papers. Time constants $\tau_{k d}, \tau_{d}, \tau_{k}$ is determinated by the intersection between the slope dv/dt and EMF is time

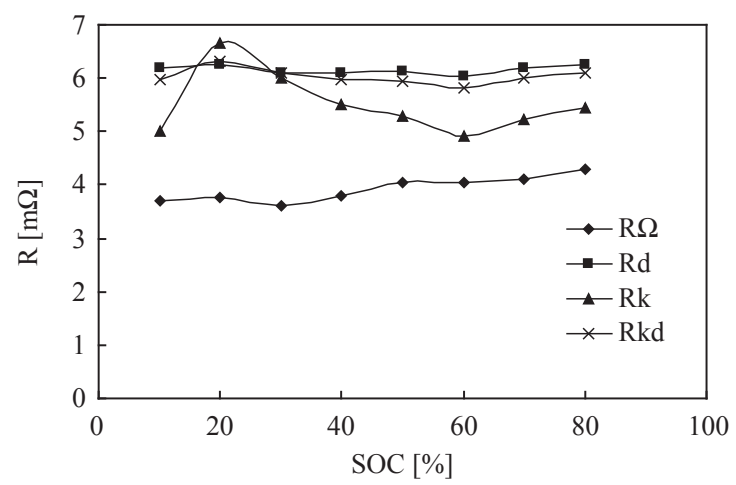

Fig. 7 Relationship of $R_{\Omega}, R_{k d}, R_{d}, R_{k}$ and SOC under discharge

constant [Schweighofer et al., 2003; Tian et al., 2009]. Figure 7 are relationship of $R_{\Omega}, R_{k d}, R_{d}, R_{k}$ and SOC under discharge.

\section{PARAMETERS IDENTIFICATION OF EQUIVALENT CIRCUIT MODE 6.1 Discharge}

Put these six parameters $R_{\Omega}, R_{k d}, R_{d}, R_{k}, I, \tau_{d}, \tau_{k}, \tau_{k d}$ into (4), (7), and (10), SOC under four states of discharge/charge can be calculated. Figure 8 and Figure

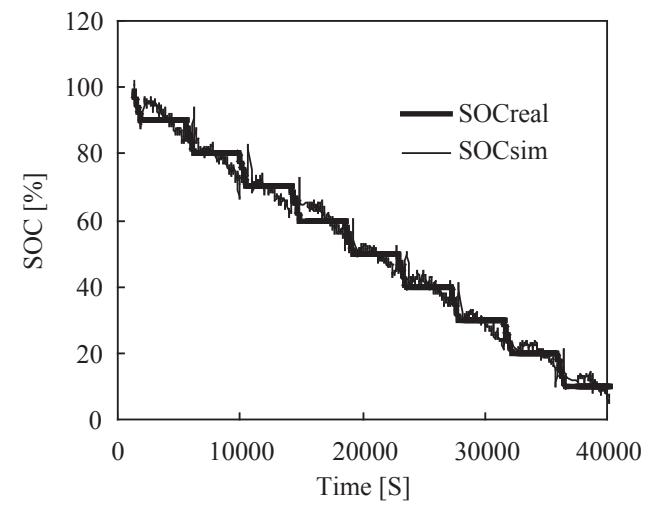

Fig. 8 SOC comparison between experimental results and first order model calculated data under discharge

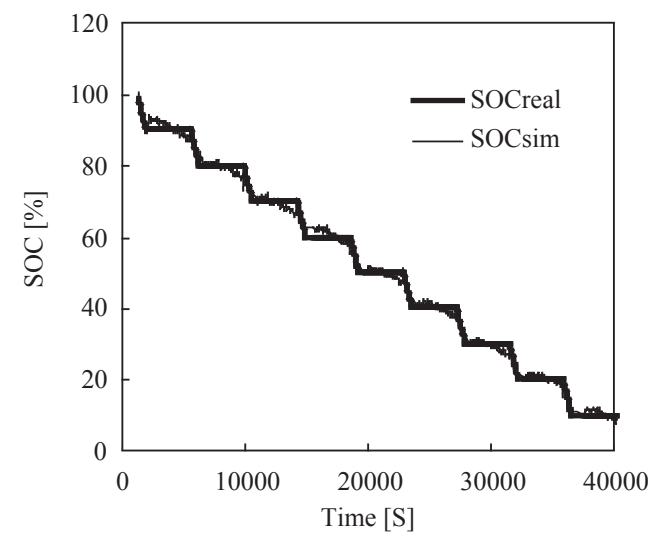

Fig. 9 SOC comparison between experimental results and second order model calculated data under discharge 


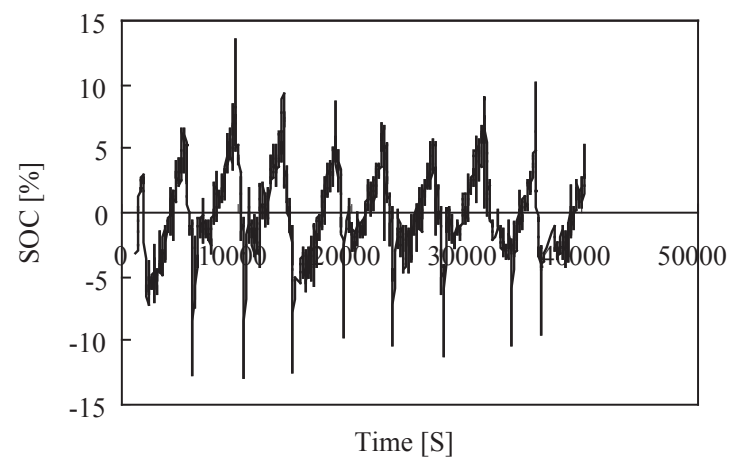

Fig. 10 Error of first order model calculated data under discharge

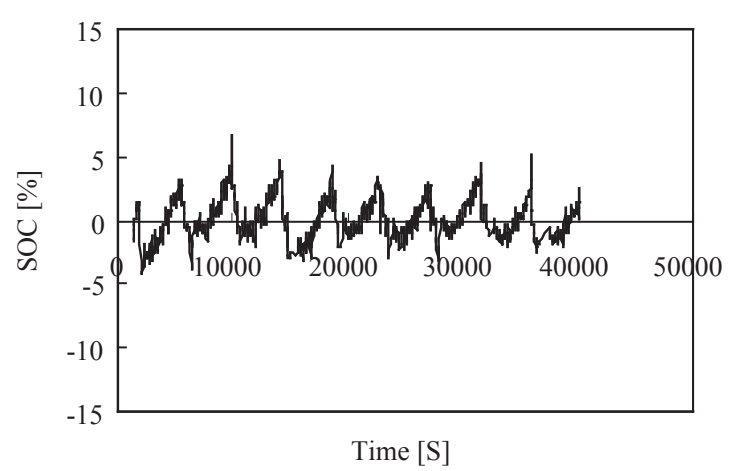

Fig. 11 Error of second order model calculated data under discharge

9 show the comparison between experiment data (blue) and calculated data (pink). Figure 10 and Figure 11 show two errors of proposed method used in the first order and the second order models. The results show that precisions of the second order and the first order are good. Error of second model is less than $7 \%$, and error of the first model is less than $15 \%$. Therefore, the second order model is suit for high precise requirement system and the first order model is easy to implement.

\subsection{Charge}

Put these six parameters $R_{\Omega}, R_{k d}, R_{d}, R_{k}, I, \tau_{d}, \tau_{k}, \tau_{k d}$ into (8), (9) and (10), SOC under four states of discharge/charge can be calculated. Figure 12 and Figure 13 show the comparison between experiment data (blue) and calculated data (pink). Figure 14 and Figure 15 show two errors of proposed method used in first order and second order models. The results show that precisions of second order and first order are good. Error of second model is less than $9.5 \%$, and error of first model is less than $19 \%$. Therefore, second order model is suit for high precise requirement system and first order model is easy to implement.

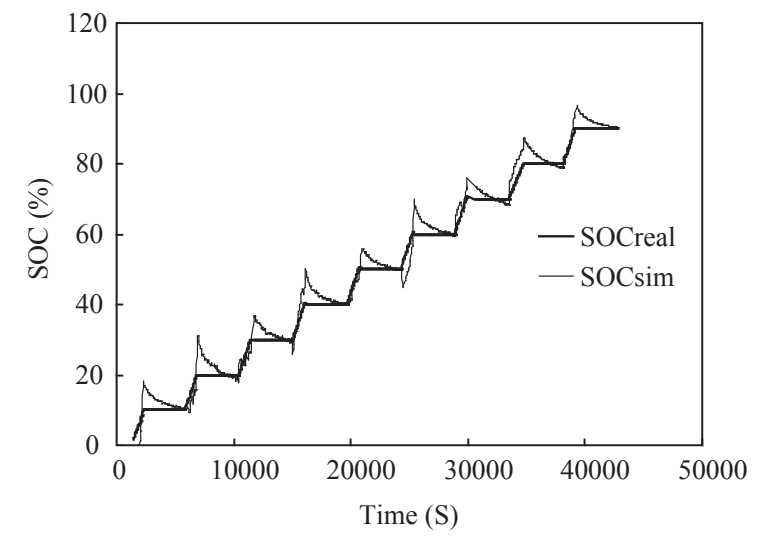

Fig. 12 SOC comparison between experimental results and first order model calculated data under charge

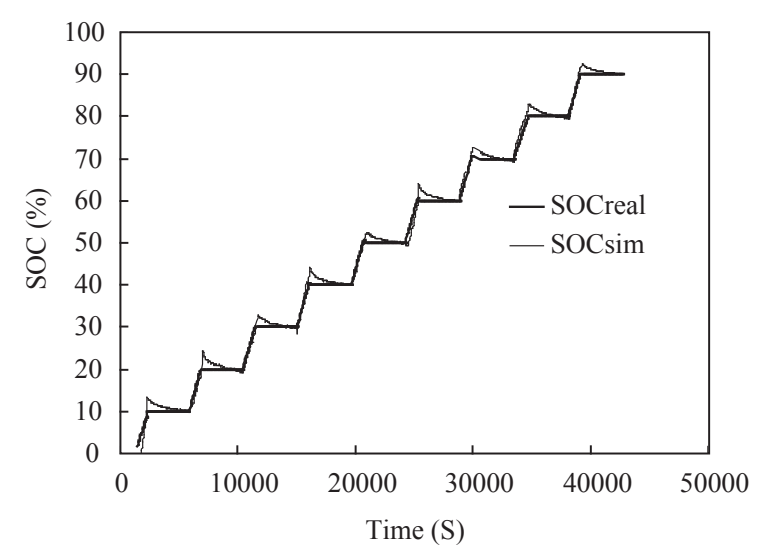

Fig. 13 SOC comparison between experimental results and second order model calculated data under charge

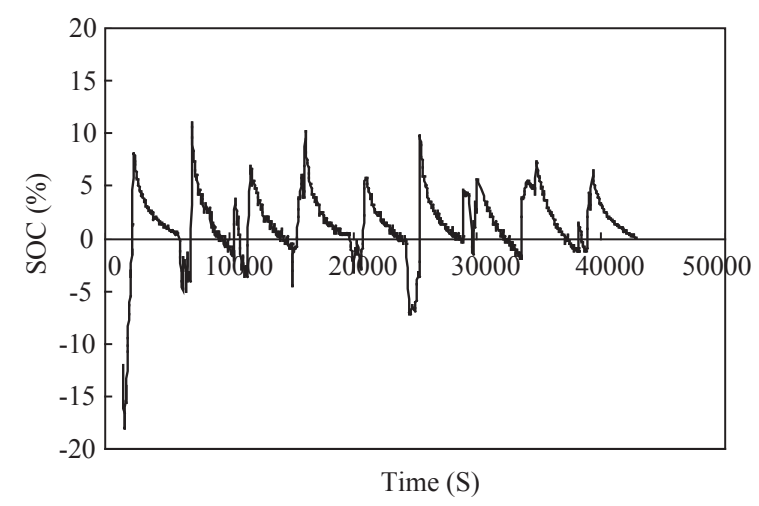

Fig. 14 Error of first order model calculated data under charge

\section{CONCLUSION}

A piece-wise relationship between SOC and EMF was achieved by pulse discharge experiment. By means of first order and second order equivalent circuit model analysis, four states of the battery charge/discharge 


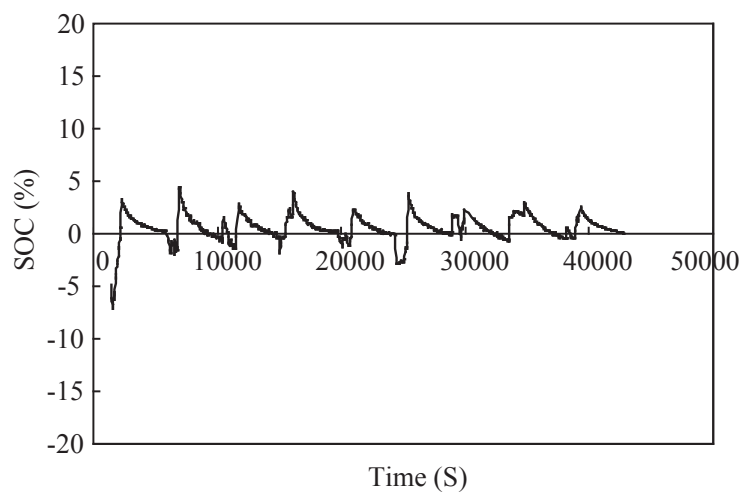

Fig. 15 Error of second order model calculated data under charge

phase were discussed; moreover, physical meaning of capacitor and resistance branch was introduced. The relationship among the battery terminal Voltage, model parameters and SOC was set.

Comparison between experimental data and calculated data based on proposed method was undertaken. The precise of the first order model is less than 19 percent, and the precision of the second order model is less than 9.5 percent. The result shows the precision of proposed method is good and the precision of second order model is higher than first order model.

\section{References}

Barbarisi, O., R. Canaletti, and L. Glielmo, State of charge estimator for NiMH batteries, Proceeding of 41st IEEE Conference on Decision and Control, 1739-1744, 2002.

Chan, C. C., E. W. C. Lo, S. Weixiang, The new calculation approach of the available capacity of batteries in electric vehicles, Proceedings of EVS 17, CD-Rom, 2000.

Hammouche, A., E. Karden, R. W. D. Doncker, Monitoring state-of-charge of Ni-H and Ni-Cd batteries using impedance spectroscopy, Journal of Power Sources, Vol. 127, No. 1-2, 105-111, 2004.

Hansen, T., and C. J. Wang, Support vector based the battery state of charge estimator, Journal of Power Sources, Vol. 141, No. 2, 351-358, 2005.

James, H. A., A. Thieme, W. Barry, and A. Johnson, A battery state-of-charge indicator for electric wheelchairs, IEEE Transactions on Industrial Electronics, Vol. 39, No. 5, 398-409, 1992.

Jung, D. Y., B. H. Lee, and S. W. Kim, Development of the battery management system for nickel-metal hydride batteries in Electric Vehicle applications, Journal Of Power Sources, Vol. 109, No. 1, 1-10, 2002.

Koehler, U., and C. Schmitz, Nickel metal hydride batteries for hybrid vehicles and new vehicle power supply systems, Proceedings of EVS 18, CD-Rom, 2001.

Kremer, P., and G. Bopp, State of charge display for lead-acid batteries, intention, methods, reality, Proceedings of 13th European PhotoVoltaic Solar Energy Conference, 1763-1769, 1995.

Onishi, M., T. Miyata, H. Goto, H. Sonobe, M. Isogai, A. Emori, T. Kinoshita, and M. Nakanishi, Ni/MH battery system for HEV applications, Proceedings of EVS 18, CD-Rom, 2001.

Salkind, A. J., C. Fennie, P. Singh, T. Atwater, and D. Reisner, Determination of state-of-charge and stateof-health of batteries by fuzzy logic methodology, Journal of Power Sources, Vol. 80, 293-300, 1999.

Schweighofer, B., K. M. Raab, and G. Brasseur, Modeling of high power automotive batteries by the use of an automated test system, IEEE Transactions on Instrumentation and Measurement, Vol. 52 No. 4, 1087-1091, 2003.

Singh, P., C. Fennie, and D. Reisner, Fuzzy logic modeling of state-of-charge and available capacity of nickel/metal hydride batteries, Journal of Power Sources, Vol. 136, No. 2, 322-333, 2004.

Singh. P., and D. Reisner, Fuzzy logic-based state of health determination of lead acid batteries, Proceedings of Telecommunications Energy Conference, 583-590, 2002.

Stiegeler, M., T. Frey, S. Rohr, and H. Kabza, The battery management system for lead acid battery with calibration using charge and discharge rest voltage characteristic, Proceedings of EVS 21, CD-Rom, 2005.

Tian, S., M. Hong, and M. Ouyang, An experimental study and nonlinear modeling of discharge I-V behavior of value-regulated lead-acid batteries, IEEE Transactions on Energy Conversion, Vol. 24 No. 2, 452-458, 2009.

Youn, K., C. Kim, and J. Koo, Development of the battery system for Hyundai HEV, Proceeding of EVS21, CD-Rom, 2005.

(Received March 20, 2010; accepted May 1, 2010) 